\title{
Warfarin Therapy That Results in an International Normalization Ratio above the Therapeutic Range Is Associated with Accelerated Progression of Chronic Kidney Disease
}

\author{
Sergey V. Brodsky ${ }^{a}$ Michael Collins ${ }^{b}$ Edward Park ${ }^{b}$ Brad H. Rovin $^{b}$ \\ Anjali A. Satoskar ${ }^{a}$ Gyongyi Nadasdy ${ }^{a}$ Haifeng Wu ${ }^{a}$ Udayan Bhatt ${ }^{b}$ \\ Tibor Nadasdy ${ }^{a}$ Lee A. Hebert ${ }^{b}$
}

Departments of a Pathology and ${ }^{\mathrm{b}}$ Medicine, The Ohio State University, Columbus, Ohio, USA

\section{Key Words}

Warfarin · Serum creatinine $\cdot$ Acute kidney injury $\cdot$ Chronic kidney disease

\begin{abstract}
Background/Aims: We had previously reported that acute kidney injury (AKI) in warfarin-treated chronic kidney disease (CKD) patients may occur shortly after an acute increase in the International Normalization Ratio (INR) >3.0 with formation of occlusive red blood casts. Recovery from this warfarin-associated AKI is poor. Here we investigated whether excessive warfarin therapy could accelerate the progression of CKD. Methods: We analyzed serum creatinine (SC) and INR in 103 consecutive CKD patients on warfarin therapy in our Nephrology program from 2005 to the present. Results: Forty-nine patients experienced at least 1 episode of INR $>3.0$. Of these, 18 patients (37\%, Group 1) developed an unexplained increase in SC $\geq 0.3 \mathrm{mg} / \mathrm{dl}$ coincident with INR $>3.0$ (mean SC increase $0.61 \pm 0.44 \mathrm{mg} / \mathrm{dl}) ; 31$ patients $(63 \%$, Group 2) showed stable SC (mean SC change $0.04 \pm 0.19$ $\mathrm{mg} / \mathrm{dl})$. Subsequent CKD progression was accelerated in Group 1, but not in Group 2. The 2 groups were not different with respect to demographics, comorbidities, blood pressure, or therapies. However, African Americans were over-
\end{abstract}

represented in Group 1 ( $p=0.035)$. Conclusions: Overanticoagulation is associated with faster progression of CKD in a high percentage of patients. Our results indicate the need for prospective trials. Nevertheless, we suggest that our findings are sufficiently compelling at this point to justify extra caution in warfarin-treated CKD patients to avoid overanticoagulation.

Copyright $\odot 2010$ S. Karger AG, Basel

\section{Introduction}

There is now credible evidence from multiple sources that severe overanticoagulation with warfarin can cause acute kidney injury (AKI) and severe acute renal failure [1-3]. Based on the kidney biopsy findings, the AKI was attributable to severe glomerular hematuria that resulted in extensive renal tubular obstruction by red blood cell casts [1-3]. Recovery of kidney function was poor. Of the 10 reported patients, only 4 experienced recovery of kidney function. Those at risk for this form of AKI appear to be those who are predisposed to glomerular hemorrhage, either because the have abnormalities of glomerular membrane basement membrane width [2-5] or chronic kidney disease (CKD) [1].

\section{KARGER}

Fax +41613061234 E-Mail karger@karger.ch www.karger.com (c) 2010 S. Karger AG, Basel

$1660-2110 / 10 / 1152-0142 \$ 26.00 / 0$

Accessible online at:

www.karger.com/nec
Sergey V. Brodsky, MD, $\mathrm{PhD}$

Department of Pathology, The Ohio State University

333 W. 10th Ave, B078 Graves Hall

Columbus, $\mathrm{OH} 43210$ (USA)

Tel. +1 614688 5831, Fax +1 614688 5889, E-Mail sergey.brodsky@ osumc.edu 


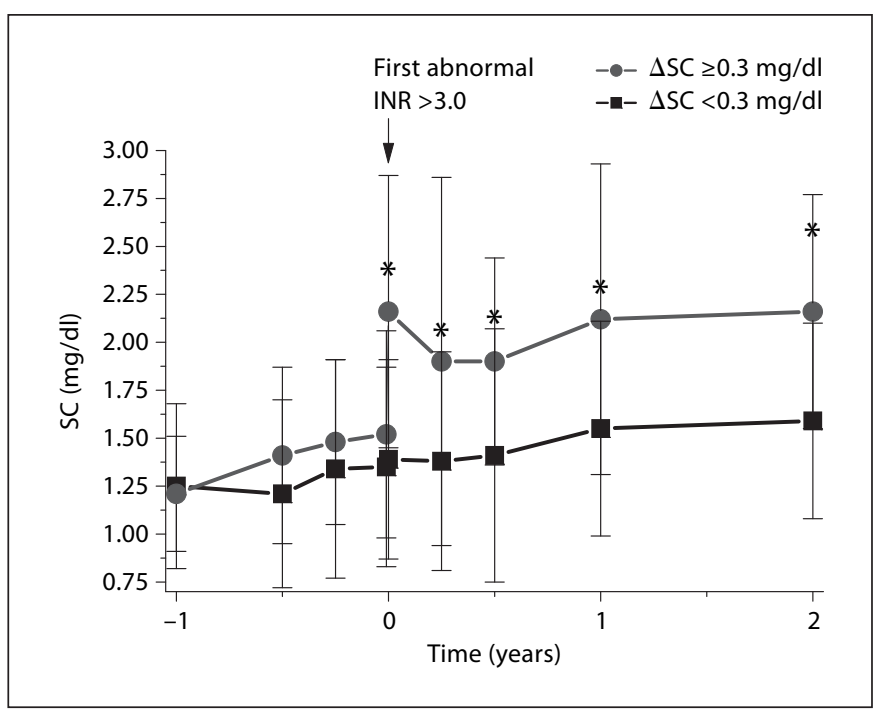

Fig. 1. Changes in the SC levels associated with an INR increase $>3.0$ IU in patients with and without accompanied AKI. Changes in the SC levels $(\triangle \mathrm{SC})$ associated with INR increase in patients with accompanied AKI $(\bullet, \mathrm{n}=18)$ and without AKI $(\boldsymbol{\square}, \mathrm{n}=31)$. The mean SC and SD are shown. ${ }^{*} \mathrm{p}<0.05$, compared to the patients without AKI. Conversion factor for SC in $\mathrm{mg} / \mathrm{dl}$ to $\mathrm{mol} / \mathrm{l}$, $\times 88.4$.

The present study assessed whether overanticoagulation with warfarin might also be a risk factor for accelerated progression of CKD. To the best of our knowledge, the present study is the first to address this question.

\section{Patients and Methods}

The present strategy was to first identify all of the CKD patients (stages 2-4) followed by our Nephrology division from 2005 to the present who received chronic warfarin therapy for any indication. This cohort consisted of 148 patients. From this cohort, we selected all of the patients who met all of the following selection criteria: (1) at least 1 episode of International Normalization Ratio (INR) $>3.0$, (2) serial measures of serum creatinine (SC) for at least 1 year before and at least 1 year after the INR $>3.0$, (3) a SC measure within 1 week of the increase in INR $>3.0$, and (4) a medical record that provided a description of their clinical status and their therapies at the time of first INR $>3.0$. This cohort consisted of 51 patients. A further exclusion criterion was a condition evident from the review of the medical record that was present at the first INR $>3.0$ that likely explained the AKI. On this basis, 2 patients were excluded because of acute onset of hypotension related to acute myocardial infarction (1 case) or acute overcontrol of blood pressure by antihypertensive medication ( 1 case).

The remaining 49 patients were stratified according to whether they experienced an unexplained increase in SC. For study purposes, this increase in SC was taken as $\geq 0.3 \mathrm{mg} / \mathrm{dl}$. This increase is more than 3 standard deviations (SDs) above the mean value for the cohort. Thus, the increase in SC is not likely to be explained by laboratory variation. Also, an acute increase in SC $\geq 0.3 \mathrm{mg} / \mathrm{dl}$ is commonly used to identify cases of AKI.

The patients who experienced a SC increase of $\geq 0.3 \mathrm{mg} / \mathrm{dl}$ were designated Group 1 . The patients who did not experience a SC increase of $\geq 0.3 \mathrm{mg} / \mathrm{dl}$ were designated Group 2 .

Clinical parameters were measured before, during, and after the episode of INR $>3.0$. The definition of 'before' is the nearest value before the date when the INR was $>3.0$, and at least within 3 months before the INR $>3.0$. The definition of 'during' (at the time of) the episode of INR $>3.0$ are measures made at, or within 1 week of the date of the INR $>3.0$.

Analytic Studies

All laboratory testing was performed in the clinical laboratories of the Ohio State University Medical Center.

\section{Statistical Analysis}

Results are presented as mean \pm SD if not otherwise specified. Differences between groups were analyzed by the two-paired $t$ test or analysis of variance test, where it was applicable. Categorical variables were analyzed by the $\chi^{2}$ contingency table analysis.

\section{Results}

Of the 148 patients initially considered for this study, 103 had serial measures of INR over a 2 -year period. Of these 49 patients, 18 (Group 1,37\%) experienced an unexplained increase in $\mathrm{SC} \geq 0.3$ at the time of the INR $>3.0$, whereas 31 patients (Group 2, 63\%) experienced no increase in $\mathrm{SC} \geq 0.3 \mathrm{mg} / \mathrm{dl}$ at the time of the INR $>3.0$.

Figure 1 shows separately the sequential mean SC values of Group 1 and Group 2 before, at the time of, and after the initial episode of INR $>3.0$. As shown, the mean acute increase in SC at the INR $>3.0$ in Group 1 was $0.61 \pm 0.44 \mathrm{mg} / \mathrm{dl}$, as compared to $0.04 \pm 0.19 \mathrm{mg} / \mathrm{dl}$ in Group 2 ( $p<0.0001)$. After the INR $>3.0$, the SC levels of Group 1 tended to remain elevated, and were significantly greater than the corresponding values of SC in Group 2.

We also assessed the changes in the SC at the first INR $>3.0$ when the SC results of Groups 1 and 2 were pooled. This analysis also showed a significant increase in the SC (from $1.42 \pm 0.53$ to $1.67 \pm 0.69 \mathrm{mg} / \mathrm{dl}, \mathrm{p}=0.048$ ). This is evidence that the significant difference in the SC at INR $>3.0$ between Groups 1 and 2 is not the result of the definition of AKI used in the study.

Table 1 shows the demographics and clinical characteristics of Group 1 and Group 2. We found a higher prevalence of African Americans among the Group 1 patients, but all other variables showed no significant differences. 
Table 1. Demographic and clinical characteristics of patients with abnormal INR episodes and increase in SC

\begin{tabular}{llll}
\hline & $\begin{array}{l}\text { Group 1 } \\
\text { (changes in } \\
\text { SC } \geq 0.3 \mathrm{mg} / \mathrm{dl} \text { ) }\end{array}$ & $\begin{array}{l}\text { Group 2 } \\
\text { (changes in } \\
\text { SC }<0.3 \mathrm{mg} / \mathrm{dl} \text { ) }\end{array}$ & p value \\
\hline Total number of patients & 18 & 31 & 0.479 \\
Age, years & $59.0 \pm 16.1$ & $62.8 \pm 18.3$ & 0.179 \\
Male/female & $11 / 7$ & $16 / 15$ & 0.035 \\
Race (w/aa/h) & $9 / 9 / 0$ & $25 / 5 / 1$ & 0.559 \\
Systolic blood pressure, mm Hg ${ }^{1}$ & $128.8 \pm 28.3$ & $123.8 \pm 12.5$ & 0.929 \\
Diastolic blood pressure, mm Hg ${ }^{1}$ & $68.7 \pm 11.5$ & $68.3 \pm 10.1$ & 0.273 \\
INR (first abnormal), IU & $4.91 \pm 3.1$ & $4.21 \pm 1.3$ & 0.354 \\
Hematuria at the time of abnormal INR (\% of patients ${ }^{1}$ ) & & & 0.957 \\
$\quad$ Negative & $50.0 \%$ & $52.6 \%$ & 0.957 \\
$\quad$ Trace (0-10 RBC/HPF) & $16.6 \%$ & $21.0 \%$ & 0.296 \\
$\quad$ Moderate (10-50 RBC/HPF) & $16.6 \%$ & $21.0 \%$ & 0.087 \\
$\quad$ Large (>50 RBC/HPF) & $16.7 \%$ & $5.3 \%$ & 0.656 \\
Treatment with warfarin recently initiated (\% of patients) & $33.3 \%$ & $12.9 \%$ & 0.180 \\
Indication for warfarin therapy (AF/APL/DVT/VR) & $11 / 2 / 5 / 0$ & $15 / 4 / 10 / 2$ & 0.460 \\
Kidney disease (\% of patients) & & & 0.717 \\
$\quad$ Diabetic nephropathy & $44.4 \%$ & $25.8 \%$ & 0.603 \\
$\quad$ HTN/renovascular diseases & $16.6 \%$ & $25.8 \%$ & $19.4 \%$ \\
$\quad$ Glomerulonephritis (membranous, SLE, IgA) & $16.6 \%$ & $29 \%$ & \\
$\quad$ Other (AKI, FSGS, nephrectomy) & $22.4 \%$ & & \\
\hline
\end{tabular}

Data are reported as mean $\pm \mathrm{SD}$ for continuous variables.

$\mathrm{w}=$ White; $\mathrm{aa}=$ African-American; $\mathrm{h}=$ Hispanic; $\mathrm{RBC}=$ red blood cells; $\mathrm{HPF}=$ high power field; $\mathrm{AF}=$ atrial fibrillation; APL = antiphospholipid syndrome; $\mathrm{DVT}=$ deep vein thrombosis or thrombosis in other vascular beds; VR = cardiac valve replacement; HTN = hypertension; SLE = systemic lupus erythematosus; IgA = IgA nephropathy; AKI = acute kidney injury; FSGS = focal segmental glomerular sclerosis.

${ }^{1}$ Records are not available for some patients.

We also assessed whether therapies at the time of the INR $>3.0$ might have differed between Group 1 and Group 2. We found that none of the following therapies differed between Groups 1 and 2: angiotensin-converting enzyme inhibitor, angiotensin receptor blocker, $\beta$ blocker, diuretic, statin, nonsteroidal anti-inflammatory drugs, aspirin, or clopidogrel.

In Group 1, the recent initiation of warfarin treatment (within the previous 3 months) tended to be more common than in Group 2 (33.3 vs. $12.9 \%, \mathrm{p}=0.087$ ). Indications for warfarin therapy are reflected in table 1 . There were no differences between Group 1 and Group 2 in the indications for warfarin therapy. The majority of patients received warfarin because of atrial fibrillation; deep vein thrombosis or thrombi in other vascular beds was the second cause of warfarin therapy. Some patients received warfarin because of antiphospholipid syndrome or a cardiac valve replacement. In addition, we did not find any difference between Group 1 and Group 2 patients with regard to the slope or intercept of the increase in SC levels versus time 1 year before warfarin therapy was begun (data not shown). Also, there was a trend towards a greater prevalence of diabetic nephropathy in Group 1 than in Group 2, although this difference did not achieve statistical significance.

To assess whether hematuria was present coincident with the acute increase in INR and SC, we searched the patient's outpatient records for urinalyses done up to 3 months before, and at the time of, the initial INR $>3.0$. About $50 \%$ of the patients in both groups had documented hematuria associated with INR $>3.0$.

With regard to comorbidities, these were no differences between Groups 1 and 2. However, advanced cardiovascular disease was prevalent in both groups. For example, severe congestive heart failure was present in 6 of 18 in Group 1 (33\%) and 12 of 31 in Group 2 (39\%). Atrial fibrillation was present at some point in 10 of 18 (56\%) in Group 1 and 16-31 (52\%) in Group 2. The indications for 


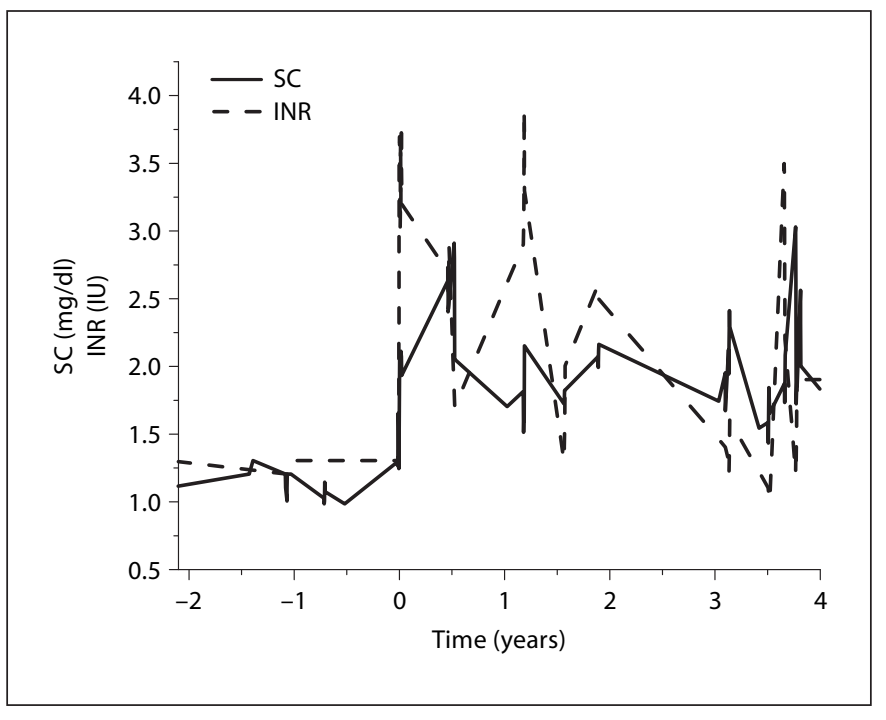

Fig. 2. Changes in SC levels associated with multiple increases in INR > 3.0 IU. Changes in SC and INR plotted for 1 patient with multiple episodes of INR >3.0 IU. Elevations in the SC levels were synchronous with abnormally high INR. There were no other associated conditions that could explain the increase in SC. Conversion factor for SC in $\mathrm{mg} / \mathrm{dl}$ to $\mathrm{mol} / \mathrm{l}, \times 88.4$.

warfarin therapy in Group 1 versus Group 2 were: atrial fibrillation ( 44 vs. $39 \%$ ), deep vein thrombosis ( 28 vs. $21 \%$ ), prosthetic heart valve (6 vs. $10 \%$ ), dilated cardiomyopathy (11 vs. $8 \%$ ), peripheral arterial disease (6 vs. $8 \%$ ), and unknown (0 vs. 5\%).

Figure 2 is a representative example of a patient who had multiple episodes of INR $>3.0$ that were accompanied by increases in SC. Figure 2 also demonstrates that although acute increases in SC tended to resolve, overall, the multiple episodes of INR $>3.0$ resulted in a sustained increase in SC, which is evidence of accelerated progression of CKD in this patient.

\section{Discussion}

The present work is a retrospective analysis of the association of SC with INR levels in warfarin-treated CKD patients. We show that in patients who experience an acute increase in INR above the therapeutic range $(>3.0)$, $37 \%$ experienced an acute increase in SC, and the SC tended to remain elevated. We hypothesize that the acute increase in SC was a consequence of warfarin overanticoagulation, based on the following: first, we have previously shown that overanticoagulation with warfarin therapy in CKD patients can cause severe AKI, which is the result of glomerular hemorrhage causing widespread tubular obstruction and impaired kidney function. We hypothesize that, in the present work, the degree of glomerular hemorrhage was less severe than that described in our previous work $[1,3]$. Second, just as in our previous work, the increase in SC in the present study was temporally related to the overanticoagulation with warfarin. Third, there were no other clinical events in these patients that could plausibly explain the increase in SC. Note that blood pressure measured at the time of INR $>3.0$ showed that the blood pressure was well controlled in both groups. Fourth, clinical follow-up and including repeat kidney biopsies from one of the patients in our original report [1] provide morphologic evidence supporting the hypothesis that continued glomerular hemorrhage, or continued effects of the red cell casts, may account for the accelerated progression of CKD. The representative patient (fig. 2) had multiple episodes of AKI associated with INR $>3.0$. Morphologic findings in consecutive kidney biopsies in this patient over an 8-month period show that coincident with the progressive rise in SC was progressive interstitial fibrosis and tubular atrophy in association with occlusive red blood cell casts in tubules.

An alternative explanation for the AKI that we observed at INR $>3.0$ is that the systemic anticoagulation could have led to spontaneous atheromatous embolism, which is a well recognized complication of systemic anticoagulation [6]. However, evidence for atheroembolization (eosinophilia, eosinophiluria, thrombocytopenia, low complement $\mathrm{C} 3$ and cutaneous or ophthalmic emboli) was not consistently searched for in our Group 1 patients, because for the most part, the AKI was unrecognized.

Previous reports have suggested that warfarin therapy can be associated with vasculitis and interstitial nephritis [7]. However, we have never observed such an association. If that occurs, it must be rare and it is unlikely to have been an important event in our Group 1 patients.

Avoiding overanticoagulation in CKD appears to be a frequent problem as described in 2 recent publications. Limdi et al. [8] compared complications of warfarin therapy among 578 patients with different stages of CKD. They reported that patients with severe CKD were at higher risk for both overanticoagulation and major hemorrhage, compared to patients with mild or moderate CKD. Chan et al. [9] reported that warfarin therapy is associated with increased mortality among hemodialysis patients than non-end-stage renal disease CKD patients. 
That study included 41,425 hemodialysis patients, of whom $8.3 \%$ were receiving warfarin therapy. Warfarin therapy was associated with a $27 \%$ increase in mortality. Our data provide an important addition to these observations.

The present work suggests that only a subset of CKD patients may be vulnerable to AKI from overanticoagulation. This is suggested by the finding that the patients with a recent administration of warfarin therapy were overrepresented in Group 1. What those risk factors for AKI might be in Group 1 versus Group 2 patients is not clear. It is noteworthy that congestive heart failure and atrial fibrillation were common in both Group 1 and Group 2 and the indications for warfarin were similar in Groups 1 and 2. The search for risk factors for warfarininduced AKI would certainly be a major focus of a prospective study of the association of chronic warfarin therapy with acute and chronic kidney injury in CKD patients.

The hypothesis underlying this study was that we would find that overanticoagulation with warfarin would adversely affect progression of CKD in a small percentage of patients. Thus, the fact, that a high percentage of our patients experienced an acute increase in SC, was unexpected. Nevertheless, because these findings are based on a rigorous and meticulous retrospective evaluation of patients who are generally well known to us, we suggest that the present work accurately describes a new and important risk factor for progression of CKD.

In summary, the present work is the first to provide evidence that chronic warfarin therapy that results in overanticoagulation is a risk factor for accelerated progression of CKD. We submit that, although the present data are compelling, it needs to be confirmed in a large prospective study which would also establish the risk factors for this association. Currently more than 2 million patients receive chronic warfarin therapy, many of whom have CKD $[10,11]$. Although the present work needs to be validated, it seems sufficiently compelling at this point to suggest that extra caution is warranted in managing warfarin therapy in CKD patients in order to avoid overanticoagulation.

\section{References}

1 Brodsky SV, Satoskar A, Chen J, et al: Acute kidney injury during warfarin therapy associated with obstructive tubular red blood cell casts: a report of 9 cases. Am J Kidney Dis 2009;2:54:1121-1126.

$\checkmark 2$ Abt AB, Carroll LE, Mohler JH: Thin basement membrane disease and acute renal failure secondary to gross hematuria and tubular necrosis. Am J Kidney Dis 2000;35: 533-536.

-3 Kabir A, Nadasdy T, Nadasdy G, Hebert LA: An unusual cause of gross hematuria and transient ARF in an SLE patient with warfarin coagulopathy. Am J Kidney Dis 2004;43: 757-760.
4 Spetie DN, Nadasdy T, Nadasdy G, et al: Proposed pathogenesis of idiopathic loin painhematuria syndrome. Am J Kidney Dis 2006; 47:419-427.

5 Hebert LA, Betts JA, Sedmak DD, et al: Loin pain-hematuria syndrome associated with thin glomerular basement membrane disease and hemorrhage into renal tubules. Kidney Int 1996;49:168-173.

-6 Sheikhzadeh A, Ehlermann P: Atheromatous disease of the thoracic aorta and systemic embolism. Clinical picture and therapeutic challenge. Z Kardiol 2004;93:10-17.

\7 Kapoor KG, Bekaii-Saab T: Warfarin-induced allergic interstitial nephritis and leucocytoclastic vasculitis. Intern Med J 2008; 38:281-283.
-8 Limdi NA, Beasley TM, Baird MF, et al: Kidney function influences warfarin responsiveness and hemorrhagic complications. J Am Soc Nephrol 2009;20:912-921.

$\checkmark 9$ Chan KE, Lazarus JM, Thadhani R, Hakim RM: Anticoagulant and antiplatelet usage associates with mortality among hemodialysis patients. J Am Soc Nephrol 2009;20:872881.

10 Yanagita M: Gas6, warfarin, and kidney diseases. Clin Exp Nephrol 2004;8:304-309.

$\checkmark 11$ Kim MJ, Huang SM, Meyer UA, et al: A regulatory science perspective on warfarin therapy: a pharmacogenetic opportunity. J Clin Pharmacol 2009;49:138-146. 